Rev. salud pública. 12 (1): 1-13, 2010

Artículos Originales/Original Articles

\title{
Seguimiento de mujeres con anormalidad citológica de cuello uterino, en Colombia
}

\section{Following-up females having an abnormal Pap smear in Colombia}

Carolina Wiesner, Ricardo Cendales, Raúl Murillo, Marion Piñeros y Sandra Tovar

Instituto Nacional de Cancerología. Bogotá, Colombia. cwiesner@cancer.gov.co, acardocen@yahoo.com, rmurillo@cancer.gov.co,mpineros@cancer.gov.co, stovar@cancer.gov.co

Recibido 21 Mayo 2009/Enviado para Modificación 26 Diciembre 2009/Aceptado 14 Enero 2010

\section{RESUMEN}

Objetivo Evaluar el acceso y la oportunidad al diagnóstico y al tratamiento que tienen las pacientes con lesiones cervicales de alto grado o cáncer de acuerdo con el reporte citológico, en Colombia entre junio 2005 a junio del 2006.

Metodología Estudio retrospectivo mediante encuestas a una muestra de mujeres con anormalidad citológica residentes de cuatro departamentos de Colombia seleccionados por conveniencia en relación con diferentes tasas de mortalidad. Se realizó análisis descriptivo y se compararon las diferencias entre los departamentos. Resultados El $27 \%$ de las mujeres con lesiones de alto grado o invasoras no tuvieron acceso a alguno de los servicios diagnósticos o terapéuticos por razones de tipo administrativo de los servicios de salud, razones clínicas y culturales de las mujeres. Discusión Un elemento crítico que explica el bajo impacto en la mortalidad por cáncer de cuello uterino en la mayoría de los países de Latino América es la disociación entre actividades de tamización y las de tratamiento.

Palabras Clave: Accesibilidad a los servicios de salud, evaluación, servicios de salud para mujeres, neoplasias de cuello uterino, cribado, Colombia (fuente: DeCS, BIREME).

\section{ABSTRACT}

Objective Evaluating the opportunity and access to diagnosis and treatment for females having had an abnormal Pap smear (high-grade epithelial lesion and cervical cancer) in Colombia from June 2005 to June 2006.

Materials and Methods This was a retrospective appraisal using a semi-closed survey of females having had an abnormal Pap smear with high squamous intraepithelial lesions or cervical cancer living in four Colombian departments. These areas were conveniently selected according to their different mortality rates. A descriptive analysis was made and the departments' differences compared.

Results It was found that $27 \%$ of females having high-grade squamous intraepithelial lesion or cervical cancer had no access to any of the diagnostic or therapeutic services. Health service administration problems and clinical and cultural ones affecting the females in the study could explain such results. 
Discussion Follow-up care after abnormal cytology was very poor and could explain the lack of cervical cancer screening's impact in Colombia and in most Latin-American countries.

Key Words: Health service accessibility, evaluation study, female health services, uterine cervical neoplasm, mass-screening, Colombia (source: MeSH, NLM).

$\mathrm{E}$ n la mayoría de los países de América Latina, con excepción de Chile, México y Costa Rica, la respuesta social frente al problema de cáncer de cuello uterino no ha logrado el impacto esperado (1-3). Para que un programa de detección de cáncer cérvico uterino logre reducir la mortalidad por esta causa se deben cumplir dos premisas fundamentales (4): primero, que se cuente con las facilidades necesarias para realizar el diagnóstico de todos los casos con citología positiva y, segundo, que una vez establecido el diagnóstico, se aplique el tratamiento. De otra manera no es posible modificar la historia natural de la enfermedad y los esfuerzos, y los costos del programa de detección, resultan inútiles (5). Se espera que la mujer cumpla las visitas que se requieren para la toma de la muestra, obtención del resultado, realización de la colposcopia-biopsia y el tratamiento en un periodo máximo de tres meses. A este respecto, Chile cuenta con un sistema que permite valorar los tiempos de oportunidad en el acceso a la atención (6).

En América Latina se ha hecho un mayor énfasis en alcanzar coberturas suficientes de citologías antes que en el control de calidad o en el seguimiento de las mujeres con anormalidad citológica (7). Hoy, en la Región, se considera que la cobertura no es el un elemento que explique el bajo impacto sobre la mortalidad (7). Explican mejor este hecho los problemas relacionados con los procesos de descentralización administrativa, la falta de organización de los programas de tamización, el control de calidad, así como la difícil articulación de los servicios preventivos con los del tratamiento (8).

En Colombia, para el año 2005, el 76,5 \% de las mujeres entre 25 a 69 años se había realizado una citología en los últimos tres años (9). Sin embargo, el cáncer de cuello uterino constituye la primera causa de muerte por cáncer (10). En el año 2002 se estimaron 6815 casos nuevos (11) y se registraron 2045 muertes por esta causa con una tasa de mortalidad ajustada por edad de 10,8 por 100000 mujeres. En este país, los planes de beneficios en salud presentan diferencias notorias de acuerdo con los sistemas de aseguramiento que parecen estar afectando la continuidad de las actividades de tamizaje. Es así como los procedimientos de colposcopia-biopsia, para las mujeres pobres afiliadas al sistema 
y para las no afiliadas, solo se incluyeron como obligatorios dentro de los planes de beneficios hasta el año 2004 (12). Por otra parte, el tratamiento de las lesiones pre-neoplásicas no es de obligatorio cumplimiento para las aseguradoras de este subgrupo de población. Antes del 2004, estas mujeres debían acudir a las entidades del gobierno y solicitar un subsidio para la realización de la colposcopia-biopsia y, posteriormente, hacer el pago de una cuota de recuperación estipulada de acuerdo con el ingreso socioeconómico (13-15). Teniendo en cuenta lo anterior, el Instituto Nacional de Cancerología, ente asesor del Ministerio de la Protección Social, consideró la necesidad de evaluar el acceso y la oportunidad al diagnóstico y al tratamiento que tienen las mujeres con reporte de citologías de lesiones intraepiteliales de alto grado (LEIAG) o carcinomas infiltrantes como un elemento critico que podría estar explicando el bajo impacto en la mortalidad por cáncer de cuello uterino en el país.

\section{METODOLOGÍA}

El proyecto fue aprobado por el Comité de Ética del Instituto Nacional de Cancerología de Colombia y es parte de la iniciativa para la evaluación de las actividades de detección temprana de cáncer de cuello uterino en Colombia. Se seleccionaron por conveniencia cuatro escenarios según la mortalidad por cáncer uterino cuello uterino (16) y el cumplimiento del indicador para la toma de citologías; de esta manera se seleccionaron dos departamentos con bajas tasas de mortalidad (Boyacá, Magdalena) y dos con altas de tasas de mortalidad (Caldas y Tolima) cada par está conformado por un departamento con bajo cumplimiento de metas (Magdalena y Tolima) y uno con un adecuado cumplimiento (Boyacá y Caldas).

Se realizó un estudio retrospectivo en el que se incluyó como universo a todas las mujeres con reporte citológico de lesión intra-epitelial de alto grado LEIAG (incluye NIC II, NIC III y cáncer in situ) o de carcinoma invasor de cuello uterino (escamocelular o adenocarcinoma) generado a partir de los laboratorios ubicados en los cuatro Departamentos del estudio entre junio de 2005 y junio del 2006.

Se construyó un marco muestral de pacientes con LEIAG cáncer invasor o cáncer in situ para cada departamento a partir de los reportes citológicos de cada laboratorio registrados entre junio de 2005 y junio del 2006. Dado que resultaba muy costoso y operativamente complejo hacer un muestreo aleatorio de elementos a partir de este marco muestral, se procedió a crear una estrategia bietápica diseñada con el objetivo de poder estimar proporciones de entre el $30 \%$ y el $40 \%$, hasta en 
subgrupos del $20 \%$, con coeficientes de variación estimados de entre el $20 \%$ y el $40 \%$. De esta manera se trazó una muestra en la que en la primera etapa se seleccionaron los municipios y en la segunda etapa se seleccionaron las mujeres. En la primera etapa se diseñó un muestreo estratificado con dos estratos para cada Departamento; un estrato de municipios de inclusión forzosa y otro de municipios seleccionados mediante muestreo aleatorio simple sin reemplazo. En la segunda etapa, en el estrato de municipios de inclusión forzosa se seleccionaron las pacientes mediante un muestreo aleatorio simple sin reemplazo, mientras que en el estrato de municipios seleccionados por muestreo aleatorio simple se hizo un muestreo por conglomerados seleccionando a todas las pacientes identificadas en el marco muestral (Tabla 1).

Tabla 1. Estrategia muestral

\begin{tabular}{lcccc}
\hline & \multicolumn{2}{c}{$\begin{array}{c}\text { Primera etapa: selección de } \\
\text { municipios }\end{array}$} & \multicolumn{2}{c}{ Segunda etapa: selección de mujeres } \\
\cline { 2 - 5 } Departamento & $\begin{array}{c}\text { Municipios } \\
\text { seleccionados } \\
\text { por IF }(\mathrm{n} / \mathrm{N})\end{array}$ & $\begin{array}{c}\text { Municipios } \\
\text { seleccionados } \\
\text { por MAS }(\mathrm{n} / \mathrm{N})\end{array}$ & $\begin{array}{c}\text { Mujeres } \\
\text { seleccionadas } \\
\text { por MAS }(\mathrm{n} / \mathrm{N})\end{array}$ & $\begin{array}{c}\text { Mujeres } \\
\text { seleccionados por } \\
\text { conglomerados }(\mathrm{n} / \mathrm{N})\end{array}$ \\
\hline Boyacá & 06 -jun & $14 / 52$ & $136 / 176$ & $45 / 45$ \\
Caldas & $04-\mathrm{abr}$ & may-22 & $197 / 282$ & $28 / 28$ \\
Magdalena & $03-$ mar & oct-22 & $119 / 188$ & $57 / 57$ \\
\hline Tolima & $09-\mathrm{sep}$ & jul-45 & $223 / 430$ & $49 / 49$ \\
\hline N: tamaño del universo, $\mathrm{n}$ : tamaño de la muestra, IF: inclusión forzosa, MAS: muestreo aleatorio simple
\end{tabular}

Se calcularon los factores de expansión de acuerdo con la estrategia muestral descrita, como el inverso de la probabilidad de inclusión, para la primera y la segunda etapas y estos luego se multiplicaron para obtener el factor de expansión definitivo. Se corrigió la ausencia de respuesta de manera específica para cada etapa. Se obtuvieron estimadores de totales y de razones de acuerdo con la estrategia muestral empleada. Se presentan las tablas de resultados con los valores expandidos, acompañados del estimador del total, el de razón y los intervalos de confianza del $95 \%$ para las razones.

Para la recolección de la información se diseñó y validó una encuesta semicerrada en donde se incluyeron variables socio-demográficas, tipo de afiliación al sistema de salud, variables relacionadas con el acceso y la oportunidad a los servicios, las razones para la falta de oportunidad en cada uno de los procedimientos y el tipo de servicio utilizado para cada uno de los procedimientos; es decir si usó servicios privados o del seguro de salud. Se solicitó consentimiento verbal a las mujeres y para verificar la información dada por ellas se solicitó su autorización para consultar la historia clínica. Se registraron las fechas de los procedimientos diagnósticos y terapéuticos. Las mujeres se contactaron seis meses después de la fecha del reporte citológico, tiempo durante el cual deberían haber recibido atención. 
La información fue digitada en un módulo para la captura de los datos diseñado en FoxPro. El procesamiento de los datos fue realizado con el paquete SPSS (Statistical Package for Social Sciences, versión 11.0). Los resultados se presentan considerando los factores de expansión correspondientes a la estrategia de muestreo. El análisis descriptivo se hizo mediante proporciones para las variables categóricas, y mediante totales y promedios para las variables numéricas. Se recurrió a la estimación por intervalo para la comparación de características entre grupos.

Las mujeres que después de seis meses del reporte citológico no habían recibido algunos de los servicios de atención fueron reportadas a su respectivas aseguradoras con copia al comité de Ética e investigaciones del Instituto de Cancerología. De acuerdo con las recomendaciones del Comité se hizo seguimiento para garantizar la atención a las mujeres.

\section{RESULTADOS}

El porcentaje de ejecución alcanzado fue de 85,6 \%, en Boyacá, 85,3\% en Caldas, 73,9\% en Magdalena y 72,1\% en Tolima, es decir que, en términos globales, la muestra se cumplió en un 78,8\%. El no cumplimiento de la muestra obedeció a las siguientes causas: no se ubicó a la paciente (10,9\%); no cumplían con los criterios de inclusión $(4,7 \%)$; habían fallecido $(2,1 \%)$; rechazaron la encuesta $(0,7 \%)$ o no conocía el reporte de la anormalidad citológica en el momento de la encuesta $(2,8 \%)$.

\section{Características sociodemográficas}

Se estimó que, en un año, en los cuatro Departamentos 1834 mujeres presentaron lesiones de alto grado en el reporte de su citología. La media de edad de las mujeres fue similar en los cuatro departamentos (42 años). En Magdalena se registró el mayor porcentaje de mujeres en estrato bajo $(87,5 \%)$ con el mayor porcentaje de analfabetismo (20,5\%). Este porcentaje fue de 16,8\% en Boyacá, $13,7 \%$ en Caldas y 10,2 \% en Tolima. En Caldas, un porcentaje alto de mujeres entiende con facilidad la que lee $(74,1 \%)$ mientras en Magdalena solo el 43,1 \% lo puede hacer. Casi todas las mujeres del estudio estaba afiliada al Sistema General de Seguridad Social en Salud (SGSSS) (98\%) con diferencias en el régimen. En Boyacá, Magdalena y Tolima la mayoría de las mujeres reciben subsidio por parte del Estado, mientras que en Caldas un porcentaje superior de mujeres tienen capacidad de pago y hacen aportes al sistema de salud. 
Acceso al diagnóstico definitivo y al tratamiento

En cada uno de los departamentos un significativo porcentaje de mujeres con lesiones de alto grado no tuvieron acceso a algún tipo de servicio diagnóstico o terapéutico. En Magdalena y Tolima se observó el más alto porcentaje de pacientes a quienes no se les realizó colposcopia. En Magdalena, se presentó el más alto porcentaje de pacientes a quienes no se les realizó biopsia. La biopsia confirmó el hallazgo de una lesión de alto grado o superior en cerca del $50 \%$ de los casos para todos los departamentos excepto en Caldas en donde el porcentaje fue bastante inferior. El resultado de la biopsia no se encontró en un porcentaje relativamente importante de casos en todos los departamentos; en Boyacá y Caldas un número importante de resultados de biopsia no se pudo codificar pues el reporte no era claro en términos de contenido. El $12 \%$ de las mujeres de todos los Departamentos no tuvieron acceso al tratamiento (Tabla 2).

Si se excluyen aquellas mujeres en quienes no se pudo establecer si habían tenido acceso a alguno de los servicios, se encuentra que 486 mujeres no tuvieron acceso a los servicios, lo que representa el 26,4\% del total de mujeres (se puede calcular a partir de los datos de la Tabla 2). Los problemas más graves de acceso a alguno de los servicios se encuentran en primer lugar en Magdalena (32\%) luego Boyacá $(27,5 \%)$, Tolima (27,4 \%) y Caldas $(27,4 \%)$.

En la Tabla 3 se pueden observar las razones por las cuales las pacientes no tuvieron acceso a los procedimientos médicos. Cerca de la mitad de las mujeres de Boyacá no se realizaron los procedimientos por aspectos relacionados con sus creencias, es decir, por temor al diagnóstico, por ausencia de síntomas, porque no creen en los resultados o en los tratamientos. En Caldas y Magdalena un alto porcentaje de mujeres no se realiza la colposcopia por que el médico tuvo alguna indicación para no hacerlo. En Tolima predominaron los problemas administrativos de los servicios. La mayoría de las mujeres desconoce las razones por las cuales no se les ha iniciado tratamiento.

Oportunidad para los servicios de diagnóstico definitivo y tratamiento En la Figura 1 se muestra la mediana para la entrega de resultados de citología que muestra una oportunidad que oscila entre 19 días para Magdalena y 34 días para Boyacá. Esta gráfica se construyó solo con la información de 148 casos en Boyacá, 101 casos de Caldas, 170 casos de Magdalena y 144 casos de Tolima lo cual puede mostrar un sesgo de selección La entrega de resultados de la colposcopia es menos oportuna que la entrega de la citología con una mediana hasta de 40 días para Tolima. La entrega de resultados de la biopsia es oportuna en todos los departamentos. El mayor problema se presenta en la oportunidad para el inicio del tratamiento con una mediana similar en todos los departamentos. 


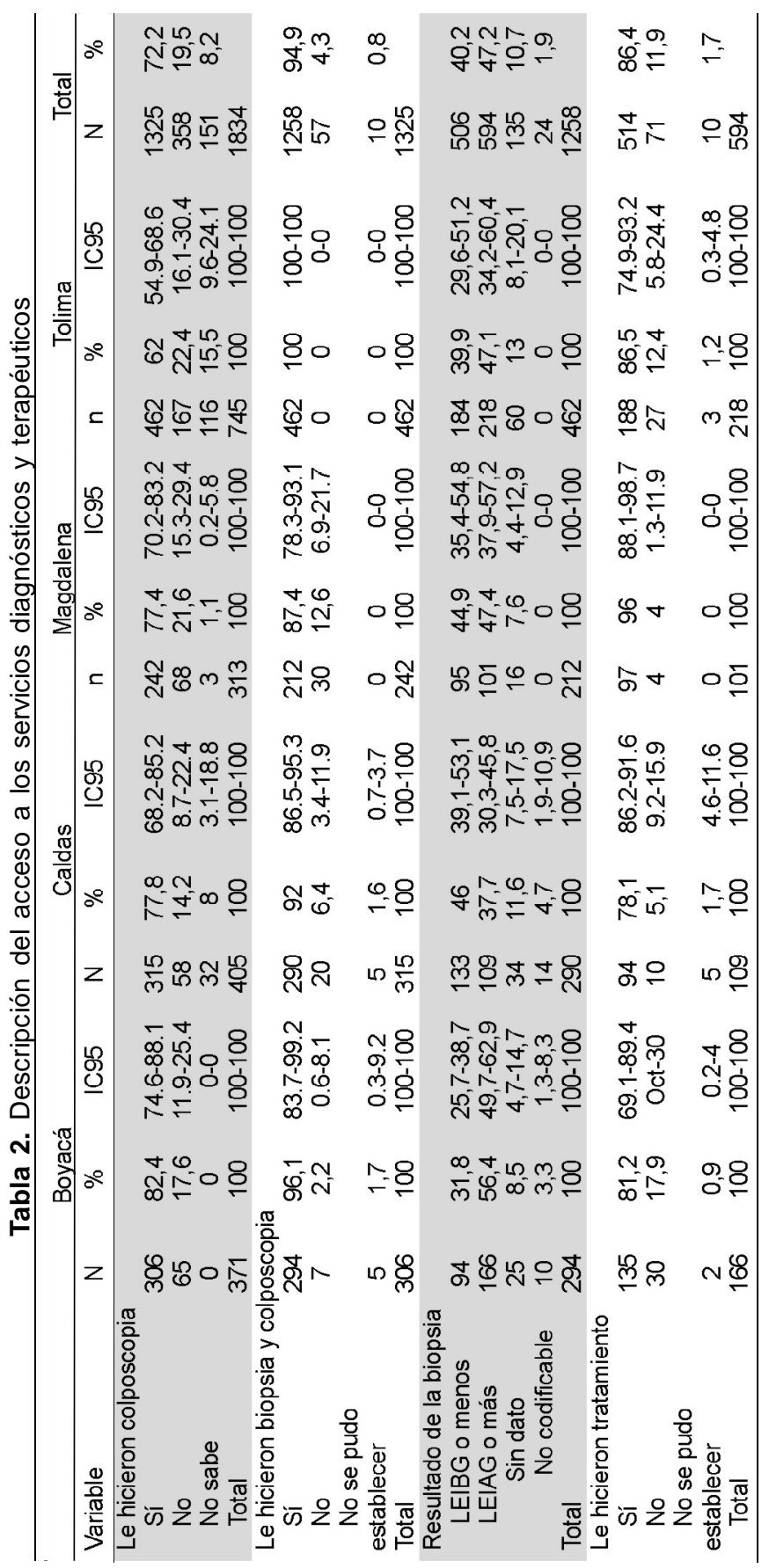


Tabla 3. Razones para la no realización de la colposcopia $v$ tratamiento

\begin{tabular}{|c|c|c|c|c|c|c|c|c|c|c|}
\hline \multirow{2}{*}{ Variable } & \multicolumn{2}{|c|}{ Boyacá } & \multicolumn{2}{|c|}{ Caldas } & \multicolumn{2}{|c|}{ Magdalena } & \multicolumn{2}{|c|}{ Tolima } & \multicolumn{2}{|c|}{ Total } \\
\hline & $\mathrm{n}$ & $\%$ & $\mathrm{n}$ & $\%$ & $\mathrm{n}$ & $\%$ & $\mathrm{n}$ & $\%$ & $n$ & $\%$ \\
\hline \multicolumn{11}{|l|}{ Razones de no realización de la colposcopia } \\
\hline No sabe, no responde, sin dato & 5 & 7,9 & 3 & 5,8 & 2 & 3 & 10 & 6,1 & 21 & 5,8 \\
\hline De tipo administrativo o & 8 & 12,5 & 5 & 8,7 & 8 & 12 & 41 & 24,6 & 62 & 17,5 \\
\hline Cambios en la atención médica & 11 & 17,1 & 22 & 38,4 & 26 & 39 & 41 & 24,6 & 101 & 28,2 \\
\hline Culturales de las mu & 31 & 47,7 & 17 & 29,3 & 20 & 30 & 41 & 24,6 & 109 & 30,6 \\
\hline Proble & 7 & 10,2 & 0 & 2,9 & 5 & 8 & 19 & 11,5 & 33 & 9,2 \\
\hline nas de saluc & 3 & 4,6 & 8 & 14,8 & 5 & 8 & 14 & 8,5 & 31 & 8,7 \\
\hline Total & 65 & 100 & 58 & 100 & 68 & 100 & 167 & 100 & 358 & 100 \\
\hline \multicolumn{11}{|l|}{ Por qué no ha iniciado el tratamiento } \\
\hline No sabe, no responde, sin dato & 8 & 27,6 & 2 & 16,7 & 0 & 0 & 19 & 71,7 & 29 & 41,2 \\
\hline ervicios & 2 & 5,1 & 3 & 33,3 & 2 & 50 & 3 & 9,4 & 9 & 13, \\
\hline Cambios en la atenc & 2 & 5,1 & 2 & 16,7 & 0 & 0 & 3 & 9,4 & 6 & 8, \\
\hline Culturales de las $\mathrm{m}$ & 7 & 22,4 & 2 & 16,7 & 0 & 0 & 0 & 0 & 8 & 11, \\
\hline Problemas económicos de las mujeres & 5 & 17,3 & 0 & 0 & 2 & 50 & 0 & 0 & 7 & 10,1 \\
\hline Otros problemas de salud o & 7 & 22,4 & 2 & 16,7 & 0 & 0 & 3 & 9,4 & 11 & 15,4 \\
\hline Total & 30 & 100 & 10 & 100 & 4 & 100 & 27 & 100 & 71 & 100 \\
\hline
\end{tabular}

Figura 1. Mediana, percentil 25 y 75 y rangos de oportunidad en días para la realización de los procedimientos de tamización, diagnóstico y tratamiento

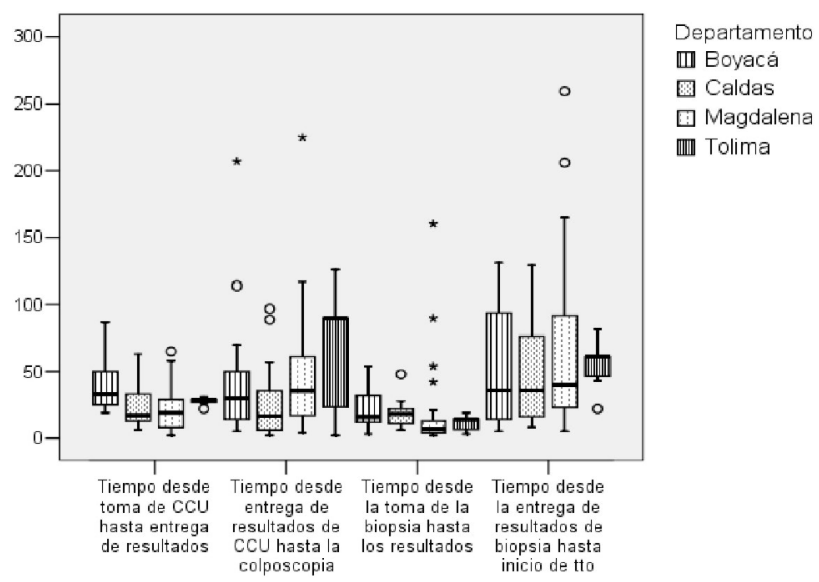

Uso de los servicios privados entre las mujeres con anormalidad citológica EL uso de los servicios privados fue relativamente importante teniendo en cuenta que se trata de mujeres de estratos bajos. Un importante porcentaje de mujeres se realizaron la citología $(12,8 \%)$, la colposcopia $(15,4 \%)$, y la biopsia $(13,9 \%)$ en servicios particulares por fuera de la red de servicios del asegurador. En Tolima un porcentaje significativamente mayor de pacientes se tomó la citología en servicios privados $(16,3 \%)$. Para la realización de la colposcopia y el procesamiento de la biopsia el uso de servicios privados es importante en tres de los departamentos con excepción del departamento Boyacá que se caracteriza por una mejor organización 
del programa de tamización (3,6 \%). En relación con el tratamiento se encuentra que el 10,8 \% de las mujeres de Tolima se realizaron el tratamiento en servicios particulares comparado con los otros departamentos en donde este porcentaje fue menor, Boyacá 1,1\%, Caldas 7,1 \%, y Magdalena 3,5\%.

\section{DISCUSIÓN}

Este trabajo presenta una descripción del acceso y la oportunidad que tienen las mujeres con citología positiva (reporte de lesión de alto grado de malignidad o cáncer) al diagnóstico definitivo y al tratamiento en cuatro departamentos de Colombia; estos departamentos se caracterizan por tener diferentes condiciones sociales y de mortalidad por cáncer de cuello uterino (16) lo cual permite tener una idea general de lo que ocurre en el país. En el estudio se encontró una disociación entre las actividades de tamización con las de diagnóstico y tratamiento pues se estima que el $27 \%$ de las mujeres con lesiones de alto grado o invasoras no culminaron el proceso hasta el tratamiento. Las razones de este hecho son por las creencias de las mujeres, por dificultades en la interpretación citológica y por problemas administrativo del sistema de salud.

Son pocos los trabajos publicados que evalúan el seguimiento a las mujeres con anormalidad citológica y dada la diversidad de situaciones descritas es difícil comparar estos hallazgos con otros estudios. En Perú (17), se identificó un pobre seguimiento en mujeres con anormalidad citológica y en Bolivia (18), se encontró que los proveedores del sector público consideran que la mayoría de las mujeres sometidas a tamizaje no regresan porque el sistema no les garantiza el diagnóstico ni el tratamiento. En México se registró una tasa acumulativa de abandono de la atención médica en 20,7 \% en mujeres con lesiones cervicales. Entre los factores que lo determinaron se encontró que la mayoría de los casos de abandono ocurrieron en la fase de diagnóstico por diferentes factores dentro de los cuales se destacaron la falta de privacidad, la falta de información y el rechazo al tratamiento (20).

El seguimiento de las mujeres con anormalidad citológica es una actividad esencial de los programas de salud pública $(21,22)$. Algunas modelaciones han mostrado que se tiene un mayor impacto sobre la reducción del riesgo de morir por cáncer cuando se hace seguimiento de mujeres con citologías positivas que cuando se hace solamente énfasis en la cobertura de tamización. (7). Por los 
hallazgos de este estudio se encuentra que en Colombia no se realiza seguimiento de las mujeres con citología positiva. Un factor que puede explicar este hecho esta dado por la orientación financiera y la planificación normativa que predomina en los sistemas de salud en Colombia y en algunos países de América Latina (8). Es decir, los procedimientos se realizan solo cuando están definidos en las normas y se pueden facturar; es así como el seguimiento a las mujeres en Colombia no se encuentra como actividad de obligatorio cumplimiento a pesar de su relevancia como actividad en salud pública.

Otra de las dificultades para hacer seguimiento es el hecho que la población de bajos recursos tiene una gran movilidad geográfica y con frecuencia carece de servicio telefónico. Además, dado que el empleo o el desempleo determinan el tipo de vínculo con el sistema de salud, se presenta una alta tasa de transición por los diferentes sistemas de aseguramiento. De acuerdo con la experiencia de otros países exitosos en el control de cáncer de cuello uterino, como es Chile, la perdida en el seguimiento de pacientes con citología anormal es un indicador que el programa ha fallado (23).

En Colombia, prácticamente la totalidad de las mujeres sabe que la citología sirve para prevenir el cáncer de cuello uterino (24). Bajo esta premisa, se espera que las mujeres a quienes se les informa que tienen una anormalidad citológica, y no tienen barreras de acceso a la red de servicios, continúen el proceso de atención hasta llegar al tratamiento. En este estudio un pequeño porcentaje de mujeres $(2,8 \%)$ no conocía el resultado de su anormalidad citológica. Este porcentaje es bajo comparado con lo registrado en otros estudios en donde se reportaron porcentajes entre $50 \%$ a $61 \%$ (20) de mujeres que no conocían el resultado de la anormalidad citológica.

En este estudio un importante porcentaje de pacientes que conocían el reporte de su anormalidad citológica tomó la decisión de no continuar con el proceso. Un elemento que explica este hecho es el peso que tiene la representación social del cáncer como sinónimo de muerte (25). El miedo a la muerte fue un factor que explicó las altas tasas de abandono en mujeres mexicanas con anormalidad citológica (20). En este sentido, resulta imperativo mejorar las estrategias de comunicación y educación a las mujeres con lesiones pre-neoplasicas de cuello uterino mediante el uso de una palabra de fácil anclaje como es pre-cáncer que las mujeres interpretan y usan adecuadamente (26) y que ya ha sido concertado y definido su adecuado uso (27). Igualmente los sistemas de salud deben facilitar al máximo el acceso a los servicios para que la mujer logre culminar su proceso terapéutico. Para ello resulta importante tener en cuenta las creencias y las condiciones psicológicas y sociales de las mujeres (28). 
Un segundo elemento que explica el no cumplimiento del proceso diagnóstico son los falsos positivos de la citología. En este estudio, $40 \%$ de las citologías reportadas como LEIAG resultaron ser lesiones de bajo grado (ASCUS) o negativas en la biopsia, mientras que no se pudo conocer el resultado de la biopsia en el $10 \%$ de los casos identificados como LEIAG. Este hecho muestra las tendencias a sobre diagnóstico de lesiones indeterminadas, el inadecuado registro de datos y la ausencia de correlación citología-biopsia que es un componente esencial del control de calidad de citologías. En Estados Unidos, el Colegio Americano de Patólogos (CAP) considera obligatoria la correlación de citología biopsia como un requisito de acreditación para todos los laboratorios de ese país (29).

Un tercer elemento que explica la falta de continuidad en la atención se debe a las características del sistema de salud. El sistema de aseguramiento fundamentado en el concepto de competencia regulada (30) ha generado dificultades en la articulación entre la financiación y la prestación de los servicios de salud (31-33). Esta se realiza en Colombia mediante la autorización de los procedimientos médicos por el paciente ante al asegurador. Este proceso se diseñó como un mecanismo para la organización de la red de servicios. Sin embargo, muchas aseguradoras convirtieron la autorización de servicios en un mecanismo de contención de costos lo cual ha tenido un impacto negativo en la oportunidad y continuidad de la atención. Este hecho ha convertido la tutela en el medio expedito para la protección del derecho a la salud (34) y al incremento en el uso de los servicios privados de atención. En Soacha, Cundinamarca, la mayoría de las mujeres se realizaban la citología en servicios privados por problemas relacionados con la calidad para la atención $(26,35)$. En el presente trabajo resulta llamativo que cerca de $15 \%$ de las mujeres pagan por procedimientos que están garantizados en los planes s de beneficios (36).

Como conclusión se encuentra que un elemento que explica el bajo impacto en la mortalidad por cáncer de cuello uterino en Colombia, es la disociación entre actividades de tamización y las de tratamiento

Agradecimientos: A las cuatro direcciones departamentales de salud, a las instituciones de salud de salud municipales e instituciones de salud, en especial a los coordinadores departamentales de las acciones para el control del cáncer de cuello uterino, Zandra Castañeda, Ruth Robles y Melva Ortiz, por facilitar el acceso a la información sobre afiliación y ubicación de las mujeres participantes en el proyecto. A las asistentes de investigación, por su labor en el trabajo de campo y la realización de las encuestas. 


\section{REFERENCIAS}

1. Palacio-Mejía LS, Rangel-Gómez G, Hernández-Avila M, Lazcano-Ponce E. Cervical Cancer, a disease of poverty: Mortality differences between urban and rural areas in México. Salud Pública de México. 2003; 45, supl 3: 315-1325.

2. Lazcano-Ponce E, Alonso P, Ruiz-Moreno JA, Hernández-Avila M. Recommendations for cervical cancer screening programs in developing countries. The need for equity and technological development. Salud Pública de México. 2003; 45, supl 3:449-461.

3. Howe SL, Vargas DE, Granada D, Smith JK. Cervical cancer prevention in remote rural Nicaragua: A program evaluation. Gynecologic Oncology, 2005; 99: S232-S235.

4. Programa de Tecnología Apropiada en la Salud (PATH). Organización Panamericana de la Salud. Planificación de programas apropiados para la prevención del cáncer cérvico uterino. $3^{\text {a }}$ edición. Washington, D.C.: OPS; 2002.

5. Lazcano-Ponce E, Alonso P, Hernández M. Cáncer cérvico-uterino diagnóstico, prevención y control.México: Editorial Médica Panamericana; 2000. p. 111.

6. República de Chile. Ministerio de Salud. Ministerio de Hacienda. Ley 19.966. por la cual se establecen las garantías explicitas en salud del régimen general de garantías. Santiago: Ministerio de Salud, Ministerio de Hacienda; 2005.

7. Murillo R, Almonte M, Pereria A, Elena F, Gamboa O, Jerónimo J, Lazcano-Ponce-E. Cervical Cancer Screening Programs in Latin America and the Carribbean. Vaccine 2008; 26s:137148.

8. Wiesner-Ceballos C, Murillo Moreno RH, Piñeros Petersen M, Tovar-Murillo SL, Cendales Duarte R, Gutiérrez MC. Control del cáncer cervicouterino en Colombia: la perspectiva de los actores del sistema de salud. Rev Panam Salud Pública. 2009; 25(1):1-8.

9. Piñeros M, Cendales R, Murillo R, Wiesner C, Tovar S. Cobertura de la citología de cuello uterino y factores relacionados en Colombia, 2005. Rev Salud Pública (Bogotá) 2007; 9(3):327341.

10. Instituto Nacional de Cancerología [Internet]. El cáncer en cifras. Disponible en: www.cancer.gov.co Consultado en: 23 de Mayo 2008.

11. Ferlay J, Bray F, Pisani P, Parkin DM. GLOBOCAN 2002 Cancer Incidence, Mortality and Prevalence Worldwide IARC CancerBase No. 5, version 2.0 IARCPress, Lyon; 2004.

12. República de Colombia. Ministerio de Salud. Consejo Nacional de Seguridad Social en Salud. Acuerdo 282 de 2004. "Por el que se fija la UPC del POS de los regímenes contributivo y subsidiados para en el año 2005 y se fijan otras disposiciones". Bogotá: Ministerio de Salud; 2004.

13. República de Colombia. Ministerio de Salud de Colombia. Decreto 2357 de 1995. Por medio del cual se reglamentan algunos aspectos del régimen subsidiado del Sistema de Seguridad Social en Salud. Bogotá: Ministerio de Salud; 1995.

14. República de Colombia. Ministerio de Salud de Colombia. Decreto 806 de 1998, artículos 32 y 33. Por el cual se reglamenta la afiliación al régimen de seguridad social en salud y la prestación de los beneficios del servicio público esencial de seguridad social en salud y como servicio de interés general, en todo el territorio nacional. Bogotá: Ministerio de Salud; 1998.

15. República de Colombia. Ministerio de la Protección social. Consejo Nacional de Seguridad Social en Salud. Acuerdo 260 del 4 de febrero de 2004. Por el cual se define el régimen de pagos compartidos y cuotas moderadoras dentro del Sistema General de Seguridad Social en Salud. Bogotá: Ministerio de Salud; 2004.

16. Murillo R, Piñeros M. Hernández G. Atlas de Mortalidad en Colombia: Bogotá: Instituto Nacional de Cancerología. Bogotá: Instituto Geográfico Agustín Codazzi; 2003.

17. Gage JC, Ferreccio C, Gonzales M, Arroyo R, Huivin M, Robles SC. Follow-up care of women with an abnormal cytology in a low-resource setting. Cancer Detect Prev 2003; 27(6):46671. 
18. Dzuba IG, Calderon R, Bliesner S, Luciani S, Amado F, Jacob M. A participatory assessment to identify strategies for improved cervical cancer prevention and treatment in Bolivia. Rev Panam Salud Publica 2005 Jul; 18(1):53-63.

19.Hernández-Alemán FR, Ornelas-Bernal LA, Apresa-García T, Sánchez-Garduño S, MartínezRodríguez OA, Hernández-Hernández DM. Motives for abandoning the healthcare process for precancerous lesions of the uterine cervix Rev Invest Clin. 2006 MayJun;58(3):217-27.

20. Population Referente Bureau. Alliance for cervical Cáncer Prevention. La prevención del cáncer cervical a nivel mundial. Washington, D.C.: PATH; 2004.

21. Alliance for Cervical Cancer Prevention. World Health Organization. International network for cancer treatment research. Planificación y aplicación de los programas de prevención y control del cáncer cérvico-uterino. Manual para gestores. Ginebra; 2006.

22. República de Chile. Ministerio de Salud. Secretaría de Atención Sanitaria, Subprograma de detección precoz de cáncer de cuello uterino. Resolución Ministerial No 480. Santiago: Ministerio de Salud; 1998.

23. Ojeda G, Ordóñez M, Ochoa L, Samper B, Sánchez F. Salud sexual y reproductiva: Resultados Encuesta Nacional de Demografía y Salud 2005. Asociación Probienestar de la Familia Colombiana (Profamilia). Profamilia, Bienestar Familiar, United State Agency for Internacional Development, Ministerio de la Protección Social; 2005.

24. Mitchell JL. Cross-Cultural Issues in the Disclosure of Cancer. Cancer Practice. 1008; 6 (3):153-160.

25. Wiesner-Ceballos C, Vejarano-Velandia M, Caicedo-Mera JC, Tovar-Murillo SL, CendalesDuarte R. [Cervical cytology in Soacha, Colombia: social representation, barriers and motivation]. Rev Salud Pública (Bogotá) 2006 Sep; 8(3):185-96.

26. Berman JJ, bores-Saavedra J, Bostwick D, Delellis R, Eble J, Hamilton SR, et al. Precancer: conceptual working definition - results of a Consensus Conference. Cancer Detect Prev 2006;30(5):387-94.

27. Fylan F. Screening for cervical cancer: a review of women's attitudes, knowledge, and behaviour British Journal of General Practice. 1998; 48, 1509-1514.

28. Zarbo RJ, Jones BA, Friedberg RC, Valenstein PN, Renner SW, Schiffmann RB, Walsh MK, et al. Q-Tracks. A College of American Pathologists Program of Continuous Laboratory Monitoring and Longitudinal Performance tracking. Arch Pathol Lab Med 2002; 126: 1036-1044.

29. Londoño JL, Frenk J. Structured pluralism: towards an innovative model for health system reform in Latin America. Health Policy 1997; 41(1): 1-36.

30. Homedes N, Ugalde A. Neoliberal reforms in health services in Latin America: a critical view from two case studies. Rev Panam Salud Publica. 2005 Mar;17(3):210-20.

31. Hernández M. Reforma sanitaria, equidad y derecho a la salud en Colombia. Cad Saude Pública. 2002;18(4):991-1001.

32. Almeida C. Reforma de sistemas de servicios de salud y equidad en América Latina y el Caribe: algunas lecciones de los años 80 y 90. Cad Saude Publica. 2002; 18(4):905-925.

33. Vélez AL. La acción de tutela: ¿un mecanismo de protección del derecho a la salud un proceso alterno para acceder a servicios de salud? Colombia Médica 2005; 36 (3): 199-208.

34. Wiesner C, Tovar S, Cendales R, Vejarano M .Organización de los servicios de salud para el control del cáncer de cuello uterino en el Municipio de Soacha. Rev Colomb Cancerol 2006; 10 (2): 98-108.

35. República de Colombia, Ministerio de Salud. Resolución No 412 del 28 de febrero de 2000. Por la cual se establecen las actividades, procedimientos e intervenciones de demanda inducida y obligatorio cumplimiento. Bogotá: Ministerio de Salud; 2000. 